\title{
In-hospital management of children with bacterial meningitis in Italy
}

Marta Ciofi degli Atti', Susanna Esposito ${ }^{2 *}$, Luciana Parola ${ }^{3}$, Lucilla Ravà', Gianluigi Gargantinii, Riccardo Longhi ${ }^{5}$ and the GSAQ working group

\begin{abstract}
Background: Over the years 2009-2013, we conducted a prospective study within a network established by the Italian Society of Pediatrics to describe the in-hospital management of children hospitalized for acute bacterial meningitis in 19 Italian hospitals with pediatric wards.

Methods: Hospital adherence to the study was voluntary; data were derived from clinical records. Information included demographic data, dates of onset of first symptoms, hospitalization and discharge; diagnostic evaluation; etiology; antimicrobial treatment; treatment with dexamethasone; in-hospital complications; neurological sequelae and status at hospital discharge. Characteristics of in-hospital management of patients were described by causative agent.

Results: Eighty-five patients were identified; $49.4 \%$ had received an antimicrobial treatment prior to admission. Forty percent of patients were transferred from other Centers; the indication to seek for hospital care was given by the primary care pediatrician in $80 \%$ of other children. Etiological agent was confirmed in $65.9 \%$ of cases; the most common infectious organism was Neisseria meningitidis (34.1\%), followed by Streptococcus pneumoniae (20\%). Patients with pneumococcal meningitis had a significant longer interval between onset of first symptoms and hospital admission. Median interval between the physician suspicion of meningitis and in-hospital first antimicrobial dose was 1 hour (interquartile range [IQR]: 1-2 hours). Corticosteroids were given to 63.5\% of cases independently of etiology; $63.0 \%$ of treated patients received dexamethasone within 1 hour of antibiotic treatment, and $41.2 \%$ were treated for $\leq 4$ days. Twenty-nine patients reported at least one in-hospital complication (34.1\%). Six patients had neurological sequelae at discharge (7.1\%). No deaths were observed.

Conclusions: We observed a rate of meningitis sequelae at discharge similar to that reported by other western countries. Timely assistance and early treatment could have contributed to the favorable outcome that was observed in the majority of cases. Adherence to recommendation for corticosteroid adjunctive therapy seems suboptimal, and should be investigated in further studies. Most meningitis cases were due to N. meningitidis and S. pneumoniae. Reaching and maintaining adequate vaccination coverage against pneumococcal and meningococcal invasive infections remains a priority to prevent bacterial meningitis cases.
\end{abstract}

Keywords: Bacterial meningitis, Children, Meningitis sequelae, Neisseria meningitidis, Streptococcus pneumoniae

\footnotetext{
* Correspondence: susanna.esposito@unimi.it

${ }^{2}$ Pediatric Highly Intensive Care Unit, Department of Pathophysiology and

Transplantation, Università degli Studi di Milano, Fondazione IRCCS Ca'

Granda Ospedale Maggiore Policlinico, Milan, Italy

Full list of author information is available at the end of the article
} 


\section{Background}

In the last 20 years, the availability of protein conjugate vaccines against Haemophilus influenzae type b (Hib), Streptococcus pneumoniae, and Neisseria meningitidis has deeply modified the epidemiology of bacterial meningitis, with a dramatic reduction in incidence documented in Countries with universal vaccination programs [1-3]. In Italy, Hib vaccination was recommended since early 1990 s, and coverage rates $>95 \%$ have been achieved [4]. Meningococcal and pneumococcal conjugate vaccines have been introduced in early 2000's, with estimated vaccination coverage rates $<70 \%$ [5]. In Italy, national surveillance of bacterial meningitis was established in 1994; in 2004, national surveillance was extended to all invasive diseases due to $\mathrm{Hib}, \mathrm{S}$. pneumoniae, and $N$. meningitidis. Over the years 2007-2012, a mean of 130 meningitis due to $N$. meningitidis and S. pneumoniae have been reported annually in children aged 0-14 years, while less than 10 cases of Hib meningitis per year were reported in the same age group [6]. National surveillance data do not provide information on in-hospital management of cases.

Early recognition of signs and symptoms suggestive of bacterial meningitis, along with prompt administration of empirical antimicrobial treatment are crucial to improve clinical outcome $[7,8]$. Timely diagnostic evaluation is also paramount to confirm etiology and modify antimicrobial treatment for optimal therapy. Guidelines for the management of bacterial meningitis in adults and children have been published $[9,10]$; however, various studies have documented criticalities in implementation of recommendations regarding diagnostic evaluation, antimicrobial and supportive treatment [11-14]. In 2008, the Study Group for Accreditation and Quality Improvement of the Italian Society of Pediatric launched a network for monitoring clinical management of children hospitalized for acute bacterial meningitis. In this article, we presented the results of the data prospectively collected by 19 Italian hospitals, from January 2009 to June 2013.

\section{Methods}

The objective of this study was to evaluate diagnostic methods and treatment of children aged 1 month to 18 years of age discharged with diagnosis of bacterial meningitis. Nineteen hospitals voluntary participated to the study; these included two tertiary care teaching children's hospitals (in northern and central Italy, respectively), three pediatric departments of tertiary-care teaching hospitals (two in northern Italy, one in southern Italy), and fourteen pediatric units of other hospitals (all in northern Italy). Bacterial meningitis was defined according to physician's clinical diagnosis at discharge, including either laboratory-confirmed or probable cases, i.e., patients with compatible signs and symptoms where cerebrospinal fluid (CSF) examination showed turbid appearance; and/or leukocytosis (>100 cells $\left./ \mathrm{mm}^{3}\right)$. Laboratory confirmation included identification of bacteria directly (by culture or polymerase chain reaction [PCR] from CSF or blood), or indirectly (by antigen detection on CSF).

This study was approved by the Ethics Committee of all the participating Centers, in compliance with the Helsinki Declaration. Written informed consent was obtained from all the parents and participants aged $\geq 8$ years gave their written assent. Information derived from clinical records included demographic data (gender, age), dates of onset of first symptoms, hospitalization and discharge; diagnostic evaluation (lumbar puncture at hospital admission, CSF formula, Gram stain, culture, latex agglutination and PCR; blood culture and PCR); etiology (confirmation of bacterial pathogen; availability of in vitro susceptibility testing within 3 days since hospital admission); antimicrobial treatment (treatment prior to admission; timing of in-hospital first dose administration, defined as interval between suspected diagnosis of meningitis and first in-hospital antibiotic administration; agents used for treatment); treatment with dexamethasone (administration, timing and duration); imaging (cranial computed tomography [CT]; cranial magnetic resonance imaging [MRI]; transfontanellar ultrasound); audiological assessment; in-hospital complications; neurological sequelae and status at hospital discharge.

Data were entered by participating hospitals in a webbased database, protected in terms of security.

To ensure completeness of data collection, hospitals reported monthly by e-mail if they did not observe cases.

All data were anonymized and exported to STATA 13.1 software (Stata Corp., College Station, TX, USA) for analysis. The database was analyzed for verifying and excluding duplicates. Proportions were calculated excluding missing values. Characteristics of hospital management of patients were described by causative agent (N. meningitidis, S. pneumoniae, other agents). Comparisons between groups were made with Chi-square or Fisher exact tests for comparing proportions of categorical variables, and with Kruskall Wallis test for comparing medians of continuous variables.

\section{Results}

\section{Patient characteristics and diagnostic evaluations}

Eighty-five patients were identified; the number of patients reported by hospital ranged from 1 to 25 , with $72.9 \%$ of total cases $(62 / 85)$ admitted to four tertiary care hospitals. Patient characteristics are shown in Table 1.

Forty percent of patients were transferred from other hospitals. 
Table 1 Characteristics of patients and diagnostic evaluation at hospital admission

\begin{tabular}{|c|c|c|c|}
\hline & \multirow[b]{2}{*}{$\mathbf{N}$} & \multicolumn{2}{|c|}{ Total $=85$} \\
\hline & & $\%$ & $95 \% \mathrm{Cl}$ \\
\hline \multicolumn{4}{|l|}{ Age } \\
\hline 1-11 months & 2 & 2.4 & $0.2-8.2$ \\
\hline $1-4$ years & 27 & 31.8 & $22.1-42.6$ \\
\hline $5-9$ years & 24 & 28.2 & $19.0-39.0$ \\
\hline $10-14$ years & 27 & 31.8 & $22.1-42.6$ \\
\hline $15-18$ years & 5 & 5.9 & $1.9-13.2$ \\
\hline \multicolumn{4}{|l|}{ Gender } \\
\hline Male & 55 & 66.3 & $53.5-74.8$ \\
\hline Female & 28 & 33.7 & $23.1-44.0$ \\
\hline \multicolumn{4}{|l|}{ Decision to seek hospital care } \\
\hline Parents & 10 & 11.8 & $5.8-20.6$ \\
\hline Transfer from other hospital & 34 & 40.0 & $29.5-51.2$ \\
\hline Primare care physician & 41 & 48.3 & $37.3-59.3$ \\
\hline \multicolumn{4}{|l|}{ Diagnostic evaluation } \\
\hline $\begin{array}{l}\text { Lumbar puncture at hospital } \\
\text { admission }\end{array}$ & 80 & 91.7 & $86.8-98.1$ \\
\hline CSF culture & 83 & 97.7 & $91.8-99.7$ \\
\hline CSF antigens & 56 & 65.9 & $54.8-75.8$ \\
\hline CSF PCR & 34 & 40.0 & $29.5-51.2$ \\
\hline Blood culture & 78 & 91.8 & $83.8-96.6$ \\
\hline Blood PCR & 28 & 32.9 & $23.1-44.0$ \\
\hline Known causative agent & 56 & 65.9 & $54.8-75.8$ \\
\hline $\begin{array}{l}\text { In vitro susceptibility testing } \\
\text { available }<\text { days since admission } \\
\text { (excluding unknown agent) }\end{array}$ & 42 & 75.0 & $38.4-60.5$ \\
\hline
\end{tabular}

95\% Cl: 95\% confidence interval; CSF: cerebrospinal fluid; PCR: polymerase chain reaction.

For patients who were not transferred from other centers, the indication to seek for hospital care was given by the primary care pediatrician in $80 \%$ of cases $(41 / 51)$.

CSF culture was performed in all patients except for two, in whom blood cultures were done. Etiological agent was confirmed in $68.2 \%$ of cases; patients without microbiological confirmation had a median CSF white blood cell count (WBC) of 530/mmc (interquartile range [IQR]: 108-4.000 WBC/mmc), with a median polymorphonuclear leukocytes (PMNs) proportion of 70\% (IQR: $60-82 \%$ PMNs) (data not shown in the table).

Interestingly, positivity rate for bacteria did not differ in patients in whom PCR testing was performed on CSF or blood (27/44; 61.3\%), compared to patients in whom only culture and no PCR testing was done (29/41; 70.7\%).

\section{Etiology and treatment}

A 7-month old infant had a nosocomial infection due to Enterococcus faecium; he was treated with teicoplanin and aminoglycosides, and had no complications or sequelae at discharge.

All the other cases were community-acquired, with onset peaking in months from October to March (54/85; 63.5\%).

The most common infectious organism was N. meningitidis, accounting for $34.1 \%$ of total cases (29 patients), followed by S. pneumoniae (20\%; 17 patients), group A Streptococcus and Streptococcus pyogenes $(5.9 \% ; 5$ patients), and Staphylococcus species (2.4\%; 2 patients) (Table 2). Hib and Mycoplasma pneumoniae were identified in one patient each.

Characteristics of patients at hospital admission and antibiotic administered by causative agent are shown in Table 2. Median interval between onset of first symptoms and hospital admission did significantly vary by causative agent; patients with meningitis due to S. pneumoniae reported a median interval of 3 days, compared to a median interval of 1 day for all other groups.

Antibiotic was given prior to admission in participating hospitals to 42 patients overall (49.4\%), with no differences by agent.

Median interval between the physician suspicion of meningitis and in-hospital first antimicrobial dose was 1 hour (IQR: 1-2 hours), independently of etiology. In vitro susceptibility testing results were available within 3 days since hospital admission for $75 \%$ of the patients with known etiological agent; in the remaining $25 \%$ of the patients, in vitro susceptibility testing were available after 4-6 days since CSF availability.

Type of antibiotic used for treatment did differ by causative agent. Third-generation cephalosporins (ceftriaxone or cefotaxime) were the only antimicrobial treatment administered to the majority of patients with confirmed N. meningitidis (62.1\%) or without etiological confirmation (55.2\%) (Table 2). Most patients with S. pneumoniae meningitis were treated with third-generation cephalosporins associated with glycopeptides (vancomicin or teicoplanin; 41.2\%), or other antibiotics (47.1\%).

Corticosteroids were given to $63.5 \%(54 / 85)$ of cases according to hospital's protocol; the proportion of children treated with dexamethasone did not significantly vary by group (Table 2). When considering timing and duration of corticosteroid administration, 63.0\% (34/54) of treated patients received dexamethasone within 1 hour of antibiotic treatment, and $41.2 \%$ (14/34) were treated for $\leq 4$ days.

\section{Other investigations, complications and outcome at discharge}

Imaging (CT scan, MRI or transfontanellar ultrasound) was performed by $64.7 \%(55 / 85)$ of patients; $48.2 \%$ underwent audiological evaluation prior to discharge, and a follow up audiological assessment was planned in 42.2\% (Table 3). Twenty-nine patients reported at least 
Table 2 Characteristics of patients at hospital admission and treatment by causative agent

\begin{tabular}{|c|c|c|c|c|c|c|}
\hline & $\begin{array}{l}\text { N. meningitidis } \\
\text { N. (29) }\end{array}$ & $\begin{array}{l}\text { S. pneumoniae } \\
\text { N. (17) }\end{array}$ & $\begin{array}{l}\text { Other } \\
\text { N. (10) }\end{array}$ & $\begin{array}{l}\text { Not known } \\
\text { N. (29) }\end{array}$ & p-value & $\begin{array}{l}\text { Total } \\
\text { N. (85) }\end{array}$ \\
\hline Median age at onset yrs - (IQR) & $4.0(1.5-7.6)$ & $1.4(0.5-4.6)$ & $0.6(0.3-7.0)$ & $5.2(0.4-8.2)$ & 0.085 & $3.3(0.5-7.1)$ \\
\hline $\begin{array}{l}\text { Median interval days between onset and hospital } \\
\text { admission - (IQR) }\end{array}$ & $1(1-2)$ & $3(2-3)$ & $1(0-2)$ & $1(1-4)$ & 0.005 & $1(1-3)$ \\
\hline $\begin{array}{l}\text { Number of patients treated with antibiotics prior to } \\
\text { hospitalization - (\%) }\end{array}$ & $14(48.3)$ & $8(47.1)$ & $2(20.0)$ & $18(62.1)$ & 0.238 & $42(49.4)$ \\
\hline $\begin{array}{l}\text { Median interval hours between suspected } \\
\text { diagnosis of meningitis and first in-hospital antibiotic } \\
\text { administration - (IQR) }\end{array}$ & $1(1-2)$ & $1(1-2)$ & $1(1-2)$ & $1(1-2)$ & 0.995 & $1(1-2)$ \\
\hline $\begin{array}{l}\text { In vitro susceptibility testing available }<3 \text { days since } \\
\text { admission, excluded unknown agent }-(\%)\end{array}$ & $22(75.9)$ & $12(70.6)$ & $8(80.0)$ & - & 0.691 & $42(75.0)$ \\
\hline Type of antibiotic administered - (\%) & & & & & $<0.001$ & \\
\hline III-generation cephalosporin & $18(62.1)$ & $2(11.8)$ & $1(10.0)$ & $16(55.2)$ & & $29(34.1)$ \\
\hline III-generation cephalosporin + glycopeptides & $3(10.3)$ & $7(41.2)$ & $1(10.0)$ & $1(3.4)$ & & $17(20.0)$ \\
\hline III-generation cephalosporin + other antibiotics & $8(27.6)$ & $8(47.1)$ & $5(50.0)$ & $11(37.9)$ & & $10(11.8)$ \\
\hline Other & $0(0.0)$ & $0(0.0)$ & $3(30.0)$ & $1(3.4)$ & & $29(34.1)$ \\
\hline Number of patients treated with dexamethasone - (\%) & $15(51.7)$ & $14(82.4)$ & $6(60.0)$ & $19(65.5)$ & 0.222 & $54(63.5)$ \\
\hline $\begin{array}{l}\text { Number of patients who received dexamethasone } \\
\text { within } 1 \text { hour of } \\
\text { antibiotic - (\%) }\end{array}$ & $9(31.0)$ & $10(58.8)$ & $5(50)$ & $10(34.5)$ & 0.544 & $34(40)$ \\
\hline $\begin{array}{l}\text { Median duration days of dexamethasone } \\
\text { treatment - (IQR) }\end{array}$ & $6(4-12)$ & $4(4-12)$ & $7(4-11)$ & $6(4-10)$ & 0.951 & $6(4-12)$ \\
\hline
\end{tabular}

IQR: interquartile range.

one in-hospital complications, including 6 seizures, 5 disseminated intravascular coagulation (DIC) cases, 3 cases with septic shock, 1 cerebral hemorrhages, and 1 syndrome of inappropriate antidiuretic hormone secretion (SIADH). Length of stay was significantly different by groups; it was longest for pneumococcal meningitis (median: 16 days), and shortest for meningococcal meningitis (median: 11 days). Status at hospital discharge was available for all patients; six patients had neurological sequelae (7.1\%). These included three children with hypoacusia, two requiring anticonvulsant therapy after a subdural hygroma, one with hydrocephalus. Patients with pneumococcal meningitis had a higher frequency of neurological sequelae $(3 / 17 ; 17.6 \%)$, than all other patients $(3 / 68 ; 4.4 \%)$, with a relative risk of 4.0 (95\% CI: 0.9 -18.1; $\mathrm{p}=0.057)$. No further factor or feature predictive of sequelae was identified. No deaths were observed.

\section{Discussion}

This study focused on clinical management of bacterial meningitis in children from a western Country, and was based on a series of cases prospectively collected in the post-vaccination era. We did not observe deaths; the rate of complications at discharge was $7.1 \%$, which is lower than reported for the European Region by a

Table 3 Characteristics of in-hospital management by causative agent

\begin{tabular}{|c|c|c|c|c|c|c|}
\hline & $\begin{array}{l}\text { N. meningitidis } \\
\text { N. (29) }\end{array}$ & $\begin{array}{l}\text { S. pneumoniae } \\
\text { N. (17) }\end{array}$ & $\begin{array}{l}\text { Other } \\
\text { N. (10) }\end{array}$ & $\begin{array}{l}\text { Not known } \\
\text { N. (29) }\end{array}$ & p-value & $\begin{array}{l}\text { Total } \\
\text { N. (85) }\end{array}$ \\
\hline Median length of stay days - (IQR) & $11(9-14)$ & $16(12-25)$ & $15(9-18)$ & $13(10-17)$ & 0.006 & $13(10-16)$ \\
\hline Number of patients who underwent imaging - (\%) & $14(48.3)$ & $10(58.8)$ & $8(80.0)$ & $23(79.3)$ & 0.065 & $55(64.7)$ \\
\hline $\begin{array}{l}\text { Number of patients with in-hospital audiological } \\
\text { assessment - (\%) }\end{array}$ & $12(41.4)$ & $11(64.7)$ & $5(50.0)$ & $13(44.8)$ & 0.468 & $41(48.2)$ \\
\hline $\begin{array}{l}\text { Number of patients with planned post-discharge } \\
\text { audiological assessment - (\%) }\end{array}$ & $10(35.7)$ & $8(50.0)$ & $7(70.0)$ & $10(34.5)$ & 0.190 & $35(42.2)$ \\
\hline Number of patients with in-hospital complications - (\%) & $12(41.4)$ & $7(41.2)$ & $2(20.0)$ & $8(27.6)$ & 0.502 & $29(34.1)$ \\
\hline $\begin{array}{l}\text { Number of patients with neurological sequelae at } \\
\text { discharge - (\%) }\end{array}$ & $0(0.0)$ & $3(17.7)$ & 1 10.0) & $2(6.9)$ & 0.094 & $6(7.1)$ \\
\hline
\end{tabular}

IQR: interquartile range. 
systematic review of published papers from 1980 to 2008 on bacterial meningitis disabling sequelae [15], but higher than the rate of sequelae estimated in Greece from 1974 to 2005 [16].

Risk of complications and sequelae do vary by causative agent, being higher for meningitis due to Streptococcus pneumoniae than for other causative agents [15-18]. As expected on the basis of national vaccination coverage rates and epidemiological data, most of our cases were due to $N$. meningitidis and S. pneumoniae. S. pneumoniae cases were less frequent but more severe than other meningitis, with a $17.6 \%$ reported rate of neurological sequelae at discharge. The proportion of pneumococcal meningitis sequelae is consistent with results from meta-analysis, reporting a median of 24\% (IQR: 14-40\%).

Heptavalent pneumococcal conjugate vaccine (PCV7) has been available in Italy since 2001; the 13-valent pneumococcal conjugate vaccine (PCV13) has been introduced at the end of 2010, and it could prevent $94 \%$ of invasive infections due to S. pneumoniae [19]. Evaluation of vaccine effectiveness was beyond the objectives of this study, although in the future it will be interesting to review cases of meningitis before and after PCV13 introduction at national level. It should however be noted that in 2008, PCV7 coverage in Italy by 24 months of age was $55 \%$ on a national basis, with wide regional variations [4]; no more recent national coverage data are available. Improving pneumococcal vaccination coverage rates should be a priority at the National level, in order to prevent disabling meningitis cases as well as other invasive pneumococcal infections.

Current literature reports that complications and sequelae are less common in meningococcal meningitis $[15,18]$, and our results confirm this finding. N. meningitidis caused however the majority of our cases, as well as most of meningitis cases reported at the national level [20]. In 2008, conjugate meningococcal C (MenC) vaccine coverage was even lower than observed for PCV7, being $37 \%$ by 24 months of age, and $16 \%$ among adolescents [4]. In 2011, approximately 50\% of reported cases were due to serogroup B [20]. The availability of conjugate meningococcal $B$ vaccine, licensed in the European Union in January 2013 but recommended in Italy since 2014 only in a minority of Regions and not at a national level, can guarantee high degree of protection against meningococcal meningitis [21]. As underlined for PCV13, achieving and maintaining optimal vaccination coverage for MenC and meningococcal $\mathrm{B}$ vaccine is crucial.

In Italy, primary care is provided to children by National Health Care pediatricians and hospital emergency access is free for all individuals. In our experience, approximately $80 \%$ of patients who were not transferred from other centers sought for hospital care advised by primary care physicians and $50 \%$ had received an antimicrobial treatment prior to hospital admission. Timely assistance and early treatment could have contributed to the favorable outcome that was observed in the majority of cases.

The choice of antibiotic is an issue to consider for acute bacterial meningitis management. First line recommended treatment of suspected meningitis is cefotaxime plus amoxicillin or ampicillin for infants $<1-3$ months, and ceftriaxone for infants and children $\geq 1-3$ months [9]. Vancomycin plus a third-generation cephalosporin has been recommended as empirical treatment in Countries with prevalence of antimicrobial-resistant isolates (10), or in children who had prolonged exposure to antibiotics [9]. Italy is one of the European Countries with higher prevalence of antimicrobial-resistance; $12 \%$ of S. pneumoniae invasive isolates in 2012 were non susceptible to penicillin [22], and 5\% of isolates in years 2006-2010 were resistant or intermediate to ceftriaxone according to the CLSI breakpoints for meningitis (MIC > $1 \mu \mathrm{g} / \mu \mathrm{L})$ [23]. In our observation, a minority of S. pneumoniae cases were treated with a third-generation cephalosporin alone, while almost half of patients received an association of a third-generation cephalosporin with vancomycin or teicoplanin. We did not collect information on antimicrobial resistance, and we did not investigate how in vitro susceptibility testing results guided decision on choosing specific antimicrobial agent. However, the circulation of ceftriaxone resistant strains throughout the Country could justify the frequent use of third-generation cephalosporins associated with glycopeptides.

The rationale for use of corticosteroids in addition to antibiotics is their anti-inflammatory action. U.S. guidelines published in 2004 recommended adjunctive dexamethasone therapy in infants and children for $\mathrm{Hib}$ meningitis, because there were evidences supporting a clinical benefit when commenced with or before antibiotic therapy [10]. In 2010, UK National Collaborating Centre for Women's and Children's Health considered administration for infants or children $>$ three months with suspected or confirmed bacterial meningitis, after interpretation of CSF parameters [9]. In 2013, a systematic review showed that corticosteroids reduced mortality in patients with meningitis due to $S$. pneumoniae and reduced severe hearing loss in children with meningitis due to Hib [24]; authors concluded that data support use in children in high-income countries.

In our results, dexamethasone was administered to almost all patients with pneumococcal meningitis (82\%), and to the majority of patients with meningitis due to other agents or without known cause (59\%). The study design does not allow to explore the possible association between corticosteroids and clinical outcome, but it is possible that administration had contributed to the low 
rate of complications we have observed. When administered, dexamethasone should be given within 1 hour of first antibiotic dose, and continued for no longer than 4 days [9]. Adherence to these recommendations was suboptimal, since timing of first dose was appropriate in $63.5 \%$ of total cases and median duration of administration was 6 days. Further studies should be conducted to describe determinants for appropriateness of corticosteroid adjunctive therapy and to define an evidence-based protocol on corticosteroid use in children with bacterial meningitis.

This study has a number of limitations. In Italy there are more than 450 pediatric units; centers who participated to this study represent a small proportion of all children's hospitals and pediatric wards. The objective of the study was to evaluate clinical management of children hospitalized for acute bacterial meningitis, and we cannot infer conclusions on disease incidence and long-term outcome. Hospitals participated on a voluntary basis; a selection bias may have occurred due to participation of centers where compliance to recommendations for acute bacterial management is higher. We collected information on in-hospital treatment and patient status at discharge; the rate of sequelae at discharge may be underestimated because in-hospital audiological evaluation was not documented for all children. It is well known that neurological sequelae, such as hearing loss and cognitive difficulties, may be diagnosed post-discharge [15]. We had no long-term follow up data, but the low rate of patients for whom the audiological assessment was planned before discharging is in our experience a reason for concern.

\section{Conclusions}

In a context of high accessibility to health services, we observed a rate of meningitis sequelae at discharge which is similar to what reported by other western countries. Most meningitis cases were due to $N$. meningitidis and S. pneumoniae. Reaching and maintaining adequate vaccination coverage against pneumococcal and meningococcal invasive infections remains a national priority to prevent bacterial meningitis cases.

\section{Competing interests}

The authors declare that they have no competing interests.

\section{Authors' contributions \\ MCDA conceived the study, participated to data analysis and interpretation and wrote the article; SE participated to study design and article writing; LP participated to study design and article writing; LR performed data analysis; GG and RL conceived the study. GSAQ working group took part in designing study objective and data collection. All of the authors read and approved the final version of the manuscript.}

\section{Acknowledgements}

We wish to thank all the Health Care Workers who collected the data in participating Hospitals.

Members of GSAQ working group:
Ospedale Pediatrico Bambino Gesù: Massimiliano Raponi, Marta Ciofi degli Atti; Cl. Ped. De Marchi, Fondazione IRCCS Osp. Maggiore Policlinico, Mangiagalli e Regina Elena:Susanna Esposito, Samantha Bosis; Claudia Tagliabue; Spedali Civili Brescia: Alessandro Plebani, Alberto Arrighini; OORR Bergamo: Valentino Conter, Alberto Vitali; Ospedale di Circolo di Busto Arsizio, Saronno: Giovanni Montrasio, Elisabetta Parolo; ASL CN1 - Ospedale SS. Annunziata-Savigliano: Luigi Besenzon, Antonella Aimar; Ospedale Luigi Sacco: Gianvincenzo Zuccotti, Vania Giacomet ; Dipartimento di Pediatria - Università di Napoli "Federico II": Alfredo Guarino, Maria Luisa Spagnuolo; Azienda ULSS 4 Alto Vicentino: Massimo Bellettato; Ospedale di Melegnano: Gianluigi Gargantini, Paola Bruni; Ospedale Civile di Legnano: Alberto Flores D'Arcais, Patrizia Macellaro; Azienda Ospedaliera di Desio e Vimercate - Presidio Ospedaliero di Vimercate: Gianfilippo Rondanini, Patrizia Calzi; AO "Ospedale Civile di Legnano" Ospedale 'G.Fornaroli' Magenta: Luciana Parola, : Elisabetta Racchi; A.O. Treviglio: Luigi Gargantini, Marisa Maccherini; Azienda Ospedaliera Sant'Anna, Como:Riccardo Longhi, Maria Teresa Ortisi; Asl 14 Chioggia: Carlo Crivellaro; Ospedale San Carlo Borromeo: Alberto Podestà, Monica Tonella; Azienda Ospedaliero-Universitaria di Parma: Dodi Icilio.

\section{Author details}

'Unit of Clinical Epidemiology, Bambino Gesù Children's Hospital, Rome, Italy. ${ }^{2}$ Pediatric Highly Intensive Care Unit, Department of Pathophysiology and Transplantation, Università degli Studi di Milano, Fondazione IRCCS Ca' Granda Ospedale Maggiore Policlinico, Milan, Italy. ${ }^{3}$ Department of Pediatrics, AO Ospedale Civile di Legnano, G.Fornaroli Hospital, Magenta, Milan, Italy. ${ }^{4}$ Department of Pediatrics, Lodi Hospital, Lodi, Milan, Italy. ${ }^{5}$ Department of Pediatrics, AO Sant'Anna, Como, Italy.

Received: 25 June 2014 Accepted: 28 October 2014

Published online: 14 November 2014

\section{References}

1. Peltola $\mathrm{H}$ : Worldwide Haemophilus influenzae type $\mathrm{b}$ diseases at the beginning of the 21st century: global analysis of the disease burden 25 years after the use of the polysaccharide vaccine and a decade after the advent of conjugates. Clin Microbiol Rev 2000, 13:302-317.

2. Hsu HE, Shutt KA, Moore MR, Beall BW, Bennett NM, Craig AS, Farley MM Jorgensen JH, Lexau CA, Petit S, Reingold A, Schaffner W, Thomas A, Whitney CG, Harrison LH: Effect of pneumococcal conjugate vaccine on pneumococcal meningitis. N Engl J Med 2009, 360:244-256.

3. Trotter $\mathrm{CL}$, Chandra M, Cano R, Larrauri A, Ramsay ME, Brehony C, Jolley KA, Maiden MC, Heuberger S, Frosch M: A surveillance network for meningococcal disease in Europe. FEMS Microbiol Rev 2007, 31:27-36.

4. ICONA Working Group: ICONA 2008: national vaccination coverage survey among children and adolescents. Rapporti ISTISAN 09/29 (in Italian); 2009:viii, 118 p. Available: http://www.iss.it/binary/publ/cont/ 09_29_web.pdf. Accessed 2014 February 21.

5. Alfonsi V, D'Ancona F, Giambi C, Nacca G, Rota MC, Regional Coordinators for Infectious Diseases and Vaccinations: Current immunization policies for pneumococcal, meningococcal $C$, varicella and rotavirus vaccinations in Italy. Health Policy 2011, 103:176-183.

6. Istituto Superiore di Sanità: Dati di sorveglianza delle malattie batteriche invasive; Available at: http://www.simi.iss.it/files/Report_MBI.pdf. Accessed 4 June 2014

7. Miner JR, Heegaard W, Mapes A, Biros M: Presentation, time to antibiotics, and mortality of patients with bacterial meningitis at an urban county medical center. J Emerg Med 2001, 4:387-392.

8. Køster-Rasmussen R, Korshin A, Meyer CN: Antibiotic treatment delay and outcome in acute bacterial meningitis. J Infect 2008, 57:449-454.

9. National Collaborating Centre for Women's and Children's Health. Bacterial meningitis and meningococcal septicaemia: Management of bacterial meningitis and meningococcal septicaemia in children and young people younger than 16 years in primary and secondary care. London (UK): National Institute for Health and Clinical Excellence (NICE); 2010 (Clinical guideline; no. 102).

10. Tunkel AR, Hartman BJ, Kaplan SL, Kaufman BA, Roos KL, Scheld WM Whitley RJ: Practice guidelines for the management of bacterial meningitis. Clin Infect Dis 2004, 39:1267-1284.

11. van de Beek D, de Gans J, Spanjaard L, Vermeulen M, Dankert J: Antibiotic guidelines and antibiotic use in adult bacterial meningitis in The Netherlands. J Antimicrob Chemother 2002, 49:661-666. 
12. Cullen MM: An audit of the investigation and initial management of adults presenting with possible bacterial meningitis. I Infect 2005, 50:120-124

13. Stockdale AJ, Weekes MP, Aliyu SH: An audit of acute bacterial meningitis in a large teaching hospital 2005-10. QJM 2011, 104:1055-1063.

14. Kelly C, Sohal A, Michael BD, Riordan A, Solomon T, Kneen R, Northwest Neurological Infections Network: Suboptimal management of central nervous system infections in children: a multi-centre retrospective study. BMC Pediatr 2012, 12:14.

15. Edmond K, Clark A, Korczak VS, Sanderson C, Griffiths UK, Rudan I: Global and regional risk of disabling sequelae from bacterial meningitis: a systematic review and meta-analysis. Lancet Infect Dis 2010, 10:317-328.

16. Vasilopoulou VA, Karanika M, Theodoridou K, Katsioulis AT, Theodoridou MN, Hadjichristodoulou CS: Prognostic factors related to sequelae in childhood bacterial meningitis: data from a Greek meningitis registry. BMC Infect Dis 2011, 11:214.

17. Kim KS: Acute bacterial meningitis in infants and children. Lancet Infect Dis 2010, 10:32-42

18. Stockmann $\mathrm{Cl}$, Ampofo $\mathrm{K}$, Byington $\mathrm{CL}$, Filloux F, Hersh AL, Blaschke $\mathrm{A}$, Cowan P, Korgenski K, Mason EO, Pavia AT: Pneumococcal meningitis in children: epidemiology, serotypes, and outcomes from 1997-2010 in Utah. Pediatrics 2013, 132:421-428.

19. Azzari C, Moriondo M, Cortimiglia M, Valleriani C, Canessa C, Indolfi G, Ricci S, Nieddu F, de Martino M, Resti M, Italian group for the study of Invasive Pneumococcal Disease: Potential serotype coverage of three pneumococcal conjugate vaccines against invasive pneumococcal infection in Italian children. Vaccine 2012, 30:2701-2705.

20. European Centre for Disease Prevention and Control: Surveillance of invasive bacterial diseases in Europe, 2011. Stockholm: ECDC; 2013.

21. Vogel U, Taha MK, Vazquez JA, Findlow J, Claus H, Stefanelli P, Caugant DA Kriz P, Abad R, Bambini S, Carannante A, Deghmane AE, Fazio C, Frosch M, Frosi G, Gilchrist S, Giuliani MM, Hong E, Ledroit M, Lovaglio PG, Lucidarme J, Musilek M, Muzzi A, Oksnes J, Rigat F, Orlandi L, Stella M, Thompson D, Pizza M, Rappuoli R, et al.: Predicted strain coverage of a meningococcal multicomponent vaccine (4CMenB) in Europe: a qualitative and quantitative assessment. Lancet Infect Dis 2013, 13:416-425.

22. European Centre for Disease Prevention and Control. Antimicrobial resistance surveillance in Europe 2012: Annual Report of the European Antimicrobial Resistance Surveillance Network (EARS-Net). Stockholm: ECDC; 2012:2013.

23. Gherardi G, D'Ambrosio F, Visaggio D, Dicuonzo G, Del Grosso M, Pantosti A: Serotype and clonal evolution of penicillin-nonsusceptible invasive Streptococcus pneumoniae in the 7-valent pneumococcal conjugate vaccine era in Italy. Antimicrob Agents Chemother 2012, 56:4965-4968.

24. Brouwer MC, Mclntyre P, Prasad K, van de Beek D: Corticosteroids for acute bacterial meningitis. Cochrane Database Syst Rev 2013, 6, CD004405.

doi:10.1186/s13052-014-0087-1

Cite this article as: degli Atti et al:: In-hospital management of children with bacterial meningitis in Italy. Italian Journal of Pediatrics 2014 40:87.

\section{Submit your next manuscript to BioMed Central and take full advantage of:}

- Convenient online submission

- Thorough peer review

- No space constraints or color figure charges

- Immediate publication on acceptance

- Inclusion in PubMed, CAS, Scopus and Google Scholar

- Research which is freely available for redistribution 